\title{
Commentary: Radical ostioplasty: The way to restore flow after arterial switch operation
}

\author{
David S. Winlaw, MBBS, MD, FRACS
}

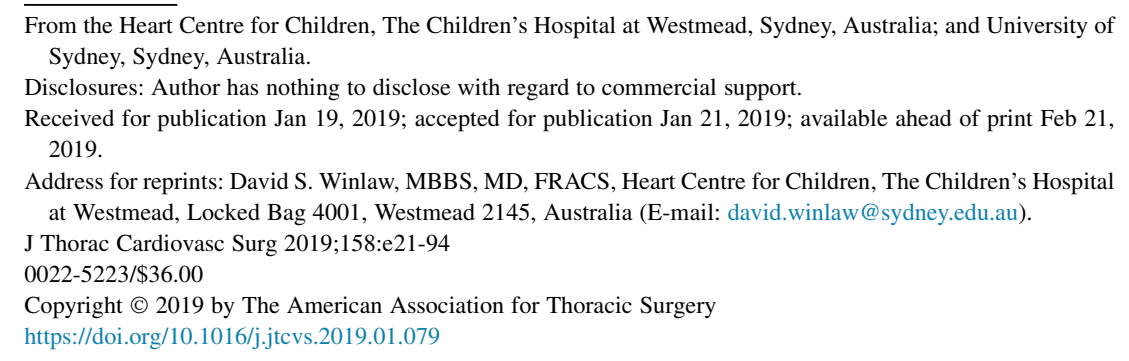

Nagato and colleagues, ${ }^{1}$ from the Mt Fuji Shizuoka Hospital group, describe an operation to address left coronary insufficiency, an unusual complication of the arterial switch operation at 4 years of age. There was evidence of collateralization of flow to the left system from a right coronary arterial injection of contrast, and the left coronary artery was noted to be small before the bifurcation into the left anterior descending and circumflex branches. The vessel was noted to be small on angiography during infancy, although it is uncertain whether this was the case at the neonatal operation or it had become smaller over time, possibly as a result of it being stretched over a dilating root.

It is remarkable how few coronary problems are evident after the arterial switch operation during the early pediatric years; successful coronary transfer with a durable outcome is presumed. This patient's caregivers did well to make a clinical diagnosis of episodic myocardial ischemia because interpreting symptoms in 4-year-olds, even with the knowledge of a previous arterial switch operation, may be difficult. Assessing the significance of coronary artery deformity on imaging and correlation of imaging with symptoms is required. Similar challenges are evident in the investigation of children for possible anomalous origin of the coronary artery from the aorta. Symptoms may be subtle until they are all too obvious and the situation is unmanageable.

The technical approach to the problem also has some aspects in common with contemporary surgery for anomalous aortic origin of the coronary artery. In conception of the operation, it is clear that the surgeons wanted to do whatever was possible to restore antegrade flow, even though this required a more complex operation with potential risks to the aortic valve and the origins of the circumflex and anterior descending branches. In discussion of such patients, whether the problem is after the arterial switch operation, anomalous origin of the coronary artery from the aorta, or even late presenting anomalous origin of the left coronary also likely to fail.

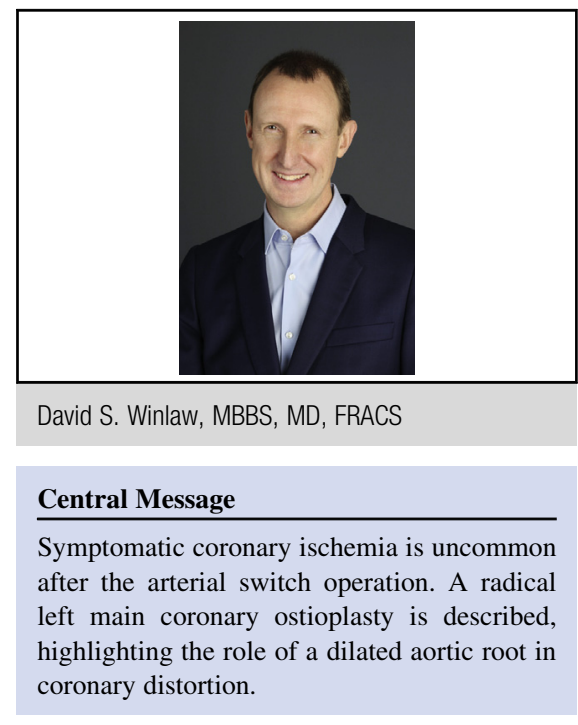

See Article page e17.

artery from the pulmonary artery, there will be advocates for the "simple" operation of grafting the left anterior descending artery. As the authors suggest, this should be reserved as a second-line approach when a radical ostioplasty has failed, because in time, particularly in the context of competitive flow from the right coronary artery, grafts are

The operation is beautifully documented, and the authors achieved a smooth angle of departure of the coronary from the aorta, a wide communication with the ascending aorta, and a shorter left main coronary with autologous pulmonary artery used to patch the ostium. As to the long term for this patient, flow might not initially be "normal," but we would expect an immediate increase in coronary perfusion pressure at the capillary level in the left coronary artery territory and that over time this antegrade pathway will grow. Ongoing monitoring, presumably with the addition of exercise testing, will be required to detect anatomic and functional limitations to flow at the reconstructed ostium.

This case gives cause to reflect on the impact of a dilating aortic root on coronary flow at later time points. This would be a concern when the coronary artery is implanted "high," which is sometimes done to avoid kinking of the implanted coronary artery. Such cases, although rare, may be present 
in subclinical forms more commonly than we suspect because of collateralization of flow and for which the described operation is an elegant solution.

\section{Reference}

1. Nagato H, Imai K, Ikai A, Sakamoto K. A novel surgical technique for the repair of coronary obstruction after arterial switch operation. J Thorac Cardiovasc Surg. 2019;158:e17-9. 